\title{
Rizotomia percutânea por radiofreqüência no tratamento das algias ororrinofaringofaciais resultantes de tumores malignos
}

\author{
Manoel Jacobsen Teixeira*, Alexandre C. do Amaral ${ }^{\star *}$, Rodrigo Labruna*** \\ Clínica Neurológica do Hospital das Clínicas da Faculdade de Medicina da USP \\ Serviço de Neurocirurgia do Hospital 9 de Julho, São Paulo, SP
}

\section{RESUMO}

São apresentados os resultados do tratamento de 39 doentes com dor orofaríngea e facial, resultante de neoplasias malignas regionais pela rizotomia percutânea por radiofreqüência do nervo trigêmeo associada, em 16 casos, à rizotomia percutânea do nervo glossofaríngeo e/ou raízes cervicais.

Ocorreram complicações em três $(7,7 \%)$ doentes. As complicações que correram de forma associada foram: paresia de motricidade ocular, disestesias focais, ceratite, úlcera de córnea e paresia mastigatória. Em três doentes, a rizotomia por radiofreqüência do nervo trigêmeo foi realizada bilateralmente. Um doente que desenvolveu dor facial por desaferentação decorrente da neuropatia actínica e da rizotomia trigeminal prévia foi submetido à nucleotratotomia espinal estereotáxica do nervo trigêmeo. Os resultados do tratamento foram excelentes em 36 casos. Ocorreu recidiva da dor em 7,7\% dos doentes. Em dois doentes, reoperação necessária foi uma nova rizotomia do nervo trigêmeo para tratamento da recorrência da dor.

Conclui-se que a rizotomia percutânea, por radiofreqüência, é um procedimento útil para o tratamento da dor orofacial decorrente do câncer. A nucleotratotomia trigeminal estereotáxica deve ser indicada como complemento à rizotomia, quando houver recidiva da dor ou ocorrência dela por desaferentação.

\section{PALAVRAS-CHAVE}

Dor oncológica. Nucleotratotomia trigeminal estereotáxica. Rizotomia percutânea por radiofreqüência.

\section{ABSTRACT}

Percutaneous rhizotomies for oropharyngofacial cancer pain The results of the treatment of 39 patients presenting oropharyngofacial pain due to cancer and treated by percutaneous trigeminal rhizotomies complemented with glossopharyngeal and or cervical rhizotomies in 16 cases are presented. In three patients the trigeminal rhizotomy was bilateral.

The results were excellent in 36 cases. Complications was observed in $7.7 \%$ of the cases. Recurrence of pain was observed in $7.7 \%$ of the cases. In two patients the trigeminal rhizotomy was repeated due to full recurrence of pain. One patient presented deafferentation pain due to trigeminal actinic neurophaty and to the previous trigeminal rhizotomy and was successfully treated with stereotactic percutaneous trigeminal nucleotractotomy.

It is concluded that percutaneous rhizotomies are efficient in the treatment of oropharyngofacial oncologic pains and that nucleotractotomy of the trigeminal caudalis nucleus is effective for deafferentation facial pain.

\section{KEYWORDS}

Oncologic pain. Percutaneous rhizotomies. Stereotactic trigeminal nucleotomy.

* Neurocirurgião, Professor Doutor do Departamento de Neurologia da Faculdade de Medicina da USP.

** Médico observador do Centro de Dor do Hospital das Clínicas da Faculdade de Medicina da USP.

***Médico colaborador do Centro de Dor do Hospital das Clínicas da Faculdade de Medicina da USP. 


\section{Introdução}

A execução de procedimentos cirúrgicos destinados ao alívio da dor é uma importante atividade dos neurocirurgiões. Dentre as algias rebeldes ao tratamento conservador, aquelas localizadas no segmento cefálico ocupam posição de destaque. Enquanto o tratamento operatório da cefaléia e de algumas variedades de dor facial é controverso e desencorajador, os procedimentos neurocirúrgicos destinados ao tratamento das neuralgias e da dor sintomática sediada na área de distribuição dos nervos cranianos e cervicais são comprovadamente eficazes ${ }^{95}$.

Várias técnicas são empregadas para o tratamento das algias orofaciais, embora nenhuma seja infalível ou desprovida de complicações. Destacam-se, entre elas: a secção ou a lise química das divisões ou ramos periféricos dos nervos trigêmeos, glossofaríngeo ou raízes cervicais; a descompressão ou compressão das raízes nervosas; a interrupção do trato espinal do nervo trigêmeo e das projeções centrais que veiculam a sensibilidade facial; a lesão de unidades psicocomportamentais; a estimulação elétrica das estruturas envolvidas na sensibilidade discriminativa da face; a infusão de fármacos analgésicos nos ventrículos encefálicos. A escolha do método cirúrgico depende das condições clínicas do doente, da natureza da afecção álgica e da experiência do neurocirurgião. Modernamente, têm sido evitadas as técnicas que, interrompendo completamente as vias sensitivas, possam gerar complicações representadas pelos neuromas de amputação, dor por desaferentação, déficits motores e anormalidades neurotróficas. Essa é a razão pela qual a rizotomia percutânea por radiofreqüência dos nervos trigêmeos, glossofaríngeo e das raízes cervicais é ainda considerada a mais eficaz e segura para o tratamento da dor facial sintomática ${ }^{95}$.

\section{Casuística e método}

Este trabalho é baseado na observação prospectiva de 39 doentes com dor facial e ou orofaríngea tratados cirurgicamente na Clínica Neurológica do Hospital das Clínicas da Faculdade de Medicina da Universidade de São Paulo e no Serviço de Neurocirurgia do Hospital Nove de Julho SA, entre abril de 1979 e junho de 1984.

Vinte e nove doentes eram do sexo masculino e dez, do sexo feminino. As idades variaram entre 21 e 80 anos; a maioria dos doentes tinha idades variando de 41 a 60 anos. O período de duração da dor variou de 3 a 12 meses. Em 21, a dor era à direita, em 15, à esquerda e, em 3, bilateral. Os diagnósticos etiológicos foram carcinoma da língua em 11 casos, carcinoma da face em 9 , carcinoma de faringe em 6 , carcinoma da cavidade oral em 5, carcinoma da laringe em 3 , carcinoma da base do crânio em 3 , condrossarcoma da mandíbula em 1 e metástase de neoplasia de mama na base do crânio em 1. A dor localizava-se no território de inervação da terceira divisão do nervo trigêmeo em 17 casos, nas segunda e terceira em 10 casos, nas três divisões em 6 , nas primeira e segunda em 3, na segunda em 2, e na primeira em 1. Em 16 doentes, havia dor concomitante no território de distribuição do nervo glossofaríngeo e das segunda e terceira raízes cervicais. Nove doentes apresentavam reflexo corneopalpebral hipoativo; 21, hipossensibilidade facial; 1 , alodinia; 9 , acometimento funcional do nervo facial e, 18, do nervo glossofaríngeo. Seis doentes, à época da rizotomia, apresentavam insuficiência respiratória, 3 , acentuada hipertensão arterial e 12 estavam acentuadamente desnutridos. Previamente, 39 haviam sido medicados com carbamazepina; 3 , com fenitoína; 30 , com fenotiazinas; 30 , com antidepressivos tricíclicos; e 14, com medicamentos morfínicos. Um doente havia sido previamente submetido à neurólise de ramos periféricos do nervo trigêmeo. O critério de indicação da cirurgia foi a presença de dor incapacitante resistente ao tratamento medicamentoso. A tomografia computadorizada do encéfalo ou a radiografia do crânio e da coluna cervical e a avaliação otorrinolaringológica foram realizadas em todos.

Foram realizadas 44 rizotomias percutâneas com radiofreqüência do nervo trigêmeo, 19 do nervo glossofaríngeo, 12 das raízes cervicais e 1 nucleotratotomia trigeminal espinal estereotáxica.

\section{Rizotomia percutânea por radiofreqüência}

Em regime ambulatorial, todos os doentes foram previamente orientados e instruídos sobre a necessidade de participarem do ato cirúrgico, informando ao cirurgião a localização da sensação parestésica desencadeada pela estimulação elétrica das estruturas nervosas e sobre a instalação dos déficits sensitivos termoinduzidos pela radiofreqüência. Foram submetidos à medicação pré-anestésica que consistiu de lorazepam ou flunitrazepam, na dose de $1 \mathrm{mg}$ a $2 \mathrm{mg}$, administradas por via oral ou intramuscular.

Sob anestesia geral proporcionada pela administração de alfadolona com alfaxolona ou tiobarbiturato sódico e associação de deidrobenzoperidol com fentanila, um eletródio, sob controle radioscópico e, à mão livre, foi introduzido, por via anterior, pelo forâmen oval, no interior do gânglio de Gasser, ou no buraco jugular ou nos forâmens da conjugação das segunda e terceira raízes cervicais. Os doentes foram, a seguir, despertados para informarem sobre a localização da sensação parestésica provocada pela estimulação das estruturas nervosas. Os geradores de estímulos e de radiofreqüência utilizados foram o modelo RFG-3A (Radionics Inc., Burlington, Massachusetts, USA) e o modelo MRFG-01B (Micromar, Diadema, São Paulo, Brasil). Quando necessário, o eletródio foi mobilizado até a sensação parestésica localizar-se no território da dor, para, então, lesão térmica, durante o período 
de 60 segundos, ser realizada. A monitorização da amperagem de corrente de lesão e da temperatura foi empregada para quantificar as lesões produzidas. As coagulações foram repetidas com intensidade e duração crescentes até que hipalgesia ou analgesia fosse gerada. Procurou-se preservar a sensibilidade táctil de todos os doentes. Quando o déficit sensitivo induzido não se localizou no território adequado, o eletródio foi reposicionado e foram produzidas novas lesões. Quando o doente suportava bem o desconforto a lesão foi realizada com intensidades de corrente gradativamente mais elevadas, sem anestesia geral; caso contrário, uma dose adicional de agente anestésico foi administrada. Em casos de rizotomia do glossofaríngeo, a ocorrência de bradicardia, durante a fase da estimulação e lesão, foi considerada parâmetro de posicionamento do eletródio. Quando a dor localizava-se no território da primeira divisão do nervo trigêmeo ou quando era bilateral, procurou-se proporcionar hipalgesia discreta para evitar-se a anestesia da córnea e o déficit da musculatura mastigatória.

Imediatamente após o procedimento, os doentes foram liberados para as atividades habituais. Quando foi induzida a hipoestesia na primeira divisão do nervo trigêmeo, foi recomendado o uso de colírio de metilcelulose durante as primeiras semanas que se seguiram ao procedimento.

\section{Nucleotratotomia trigeminal espinal estereotáxica}

O ato operatório consistiu na fixação do aparelho de TM-03B (Micromar, Diadema, São Paulo, Brasil) ao segmento cefálico e na realização de perimielografia cervical, com agente radiopaco iodado e lipossolúvel emulsificado, para visibilização da cisterna magna e da transição bulboespinal. Um eletródio de tungstênio foi introduzido na transição occipitocervical e dirigido, de baixo para cima e de fora para dentro, com inclinação de $20^{\circ}$ em relação aos planos transversal e sagital. $\mathrm{O}$ alvo estereotáxico programado situou-se $4 \mathrm{~mm}$ e $6,5 \mathrm{~mm}$ lateralmente à linha mediana, para os casos de dor no território da terceira e da primeira divisões do nervo trigêmeo, respectivamente. $\mathrm{O}$ controle da penetração do eletródio na estrutura nervosa foi realizado por meio da avaliação da impedância tecidual e, sua localização, confirmada mediante estimulação monopolar, à baixa voltagem, com estímulos de ondas quadradas de $5 \mathrm{~Hz}, 10 \mathrm{~Hz}$, $50 \mathrm{~Hz}$ e $100 \mathrm{~Hz}$. Quando a estimulação elétrica causava desconforto no local da dor referida, foram realizadas lesões por radiofreqüência, com a finalidade de proporcionar coagulação tecidual com o diâmetro de $2 \mathrm{~mm}$. Como gerador de estímulos e de radiofreqüência, foram utilizados o modelo RFG-3 (Radionics Inc., Burlington, Massachusetts, USA) e o MRFG-01B
(Micromar, Diadema, São Paulo, Brasil). Após a nucleotratotomia trigeminal, os doentes foram mantidos em repouso por 24 horas, sendo administrada dexametasona, na dose de $16 \mathrm{mg}$ ao dia, durante os primeiros cinco dias.

\section{Resultados}

Para a avaliação dos resultados, foram considerados a ocorrência de dor residual, o grau de déficit sensitivo, o aparecimento de outras disfunções neurológicas, além da hipoestesia decorrente da lesão por radiofreqüência na divisão trigeminal envolvida na dor, os efeitos tardios da desaferentação sensitiva, as complicações clínicas e a recorrência da dor.

Dos 39 doentes submetidos à rizotomia percutânea do nervo trigêmeo, 34 permaneceram sem dor e sem alterações funcionais, 3 sem dor, mas com alterações funcionais de outros nervos cranianos, e 2 melhoraram. A analgesia facial pós-operatória não acometeu outras divisões do nervo trigêmeo, além das previamente afetadas pela dor. Exceto em 2 casos que apresentaram hipalgesia em uma das divisões acometidas, nos demais, ocorreu analgesia. Dois $(5,1 \%)$ doentes apresentaram recorrência completa da dor na terceira divisão do nervo trigêmeo, 1 e 4 meses após o procedimento operatório, e foram submetidos à nova rizotomia do nervo trigêmeo que resultou em alívio da sintomatologia. Um doente apresentou recidiva parcial da dor, nas segunda e terceira divisões do nervo trigêmeo que foi controlada com medicamentos. Dois doentes apresentaram dor por desaferentação; uma, paresia da mastigação; uma, ceratite; uma, úlcera de córnea e um, acometimento da motricidade ocular extrínseca durante o período pós-operatório. O período de acompanhamento pós-operatório médio foi de 5,5 meses e variou entre 1 e 23 meses.

\section{Discussão}

Os processos neoplásicos da face, da cavidade oral e da faringe podem causar dor que, na maioria das vezes, apresenta características diferentes da dor da neuralgia do nervo trigêmeo ou do glossofaríngeo ${ }^{17}$. Metástases ósseas ou meníngeas de carcinomas, linfomas e melanomas ${ }^{34}$, schwanomas malignos, sarcomas trigeminais e infiltração das estruturas trigeminais por tumores malignos da cavidade oral e nasofaringe, podem freqüentemente causar dor no território do tronco nervoso acometido ${ }^{89}$. Os tumores malignos da base do crânio, geralmente carcinomas ou sarcomas da ororrinofaringe, podem erodir a estrutura óssea ou invadir a cavidade craniana pelos orifícios naturais, 
comprimir e destruir o gânglio de Gasser, as divisões periféricas ou a raiz do nervo trigêmeo, glossofaríngeo e raízes cervicais e originar dor, geralmente constante, acompanhada de anormalidades sensitivo-motoras e, freqüentemente, de lesões associadas de outros nervos cranianos $^{7,9}$. O desconforto causado pela neuropatia actínica e pela neuropatia tóxica decorrente da ação de quimioterápicos apresenta, também, características próprias que se superpõem às das neuropatias periféricas ${ }^{17}$. A dor decorrente da doença oncológica ororrinofaríngea freqüentemente é intensa e de difícil controle.

Em 39 doentes da presente casuística, a dor era causada por afecções neoplásicas malignas. Houve predominância dos indivíduos do sexo masculino e com idades variando entre 41 e 60 anos, o que concorda com o observado por outros autores ${ }^{17,31}$. Não há explicação para o predomínio da dor no lado direito da face. A natureza das lesões neoplásicas responsáveis pela dor coincidiu com o apresentado na literatura e justificou a predominância de ocorrência de dor na área inervada pela terceira divisão do nervo trigêmeo e, eventualmente, no território do nervo glossofaríngeo e das raízes cervicais ${ }^{17}$. Grau avançado de desnutrição causado pela neoplasia e pela dificuldade de ingestão de alimentos foi observado em $30,7 \%$ dos casos. Havia insuficiência respiratória em $15,4 \%$ dos doentes, em decorrência do bloqueio das vias aéreas superiores pela neoplasia ou pneumopatia crônica, própria dos processos neoplásicos ororrinofaríngeos.

Com finalidade analgésica, medicação psicotrópica e antiinflamatória não-esteroidal foi empregada em todos os doentes. Em 35\% dos casos, medicação morfinica havia sido previamente prescrita. Esse regime é considerado apropriado para o tratamento farmacológico da dor oncológica ${ }^{24}$. Entretanto, em casos de lesões neoplásicas da face e da cavidade orofaríngea, a dor é freqüentemente refratária ao tratamento medicamentoso ${ }^{17}$, motivo pelo qual é freqüentemente necessário o emprego de procedimentos neurocirúrgicos antiálgicos para controlá-la ${ }^{95}$.

A dor craniofacial ocorre freqüentemente em indivíduos idosos e com maior potencial de risco cirúrgico. O método operatório ideal deve eliminar a dor produzindo mínimo traumatismo das estruturas trigeminais, ser destituído de riscos e seqüelas e ter baixo índice de recorrência. Há, entretanto, muitas controvérsias a respeito dos méritos relativos dos procedimentos operatórios antiálgicos. Os procedimentos objetivando interromper as vias e as unidades sensitivas da face e orofaringe podem ser aplicados no sistema nervoso periférico (nervos trigêmeo e glossofaríngeo, raízes cervicais) e no sistema nervoso central $^{6,25,58}$.

A instilação de agentes neurolíticos nos nervos periféricos somente foi possível após o desenvolvimento das agulhas com ponta aguda e das seringas, durante a segunda metade do século XIX ${ }^{68}$. Vários agentes foram injetados nos ramos periféricos do nervo trigêmeo com finalidade analgésica: clorofórmio, ácido ósmico, álcool, cocaína com álcool etílico, cromatos, glicerina formalizada ou carbolizada, glicerina e álcool mentolado, éter, antipirina, salicilato de sódio e sais de quinino ${ }^{60,68}$. A lise dos ramos periféricos é especialmente indicada quando a dor localiza-se nas segunda e terceira divisões do nervo trigêmeo; a alcoolização da primeira divisão é difícil porque os nervos supra-orbitários são numerosos ${ }^{33}$. Apesar de a ocorrência de processos infecciosos nos tecidos transfixados pela agulha ser a única contra-indicação absoluta para a alcoolização dos ramos periféricos ${ }^{52}$, observou-se que o benefício proporcionado por tal procedimento perdurava durante poucos meses ou alguns anos ${ }^{9}$, que a repetição da neurólise era dificultada pela cicatriz provocada pelos procedimentos prévios e que a presença de proeminências ósseas na trajetória da passagem dos ramos periféricos impedia, freqüentemente, a sua realização. Com a finalidade de tornar a hipoestesia mais prolongada, substâncias neurolíticas, incluindo o álcool etílico, a uréia associada à quinina, o formaldeído, o fenol, o álcool benzílico, o fenol glicerinado, as sulfonamidas e o glicerol ${ }^{23,40,41,72}$, passaram a ser instiladas no interior do gânglio de Gasser. Essa prática tornouse popular desde o advento das agulhas longas e resistentes no início do presente século. Segundo Kubanyi ${ }^{45}$, foi Schloesser, em 1903, quem, pela primeira vez, puncionou o forâmen oval por via transoral. Segundo Penman ${ }^{68}$, Ostwalt, em 1906, empregou, pela primeira vez, a via infrazigomática para administração de agentes neurolíticos no forâmem oval e redondo. Segundo Visca e Bernasconi ${ }^{107}$, Harris, em 1909, acidentalmente, puncionou gânglio de Gasser por via lateral. Segundo Penman ${ }^{68}$, Taptas, em 1910, descreveu a técnica de punção do gânglio de Gasser por via lateral. Härtel, em 1912, descreveu a técnica de punção ganglionar por via transoval e Kirchner, em 1933, a via submentoniana. Os resultados e as complicações da gangliólise foram muito variados porque a potência dos agentes neurolíticos e a técnica empregada não foram uniformes. Apesar das modificações técnicas ${ }^{15,27,107} \mathrm{e}$ do uso de vários agentes neurolíticos ${ }^{38,67}$, a neurólise do gânglio de Gasser deixou de ser empregada, pois é pouco precisa e associa-se à elevada taxa de recorrência e de seqüelas $8,57,77,108$. Desde o fim do século passado, cirurgias visando à interrupção da raiz trigeminal, do nervo glossofaríngeo e das raízes cervicais foram desenvolvidas. Há muitas décadas, a rizotomia retrogasseriana é considerada útil para o tratamento da dor facial de natureza oncológica ${ }^{20,64}$. A rizotomia retrogasseriana subtemporal cirúrgica e a céu aberto revelou-se muito eficaz para o tratamento da dor facial oncológica $^{20,64}$. Dandy ${ }^{13}$, em 1932, recomendou a craniectomia da fossa posterior para o tratamento da dor oncológica porque, pela mesma via de acesso, é possível a secção da raiz do nervo trigêmeo do glossofaríngeo e das raízes cervicais. Dott ${ }^{14}$ recomendou, em 1951, a rizotomia e a alcoolização das estruturas trigeminais em casos de dor facial oncológica. 
As complicações e a mortalidade decorrentes dessas intervenções, especialmente em doentes debilitados ou idosos, induziram o desenvolvimento de procedimentos menos invasivos. A introdução das técnicas de rizotomia percutânea do nervo trigêmeo, glossofaríngeo e das raízes cervicais ${ }^{44,79}$ tornou o tratamento neurocirúrgico da dor facial oncológica mais seguro.

A rizotomia percutânea por radiofreqüência do nervo trigêmeo foi indicada em todos os casos da presente casuística. A dor facial oncológica, na maioria das vezes, pode ser controlada por esse procedimento ${ }^{85}$. Em nossa casuística, houve melhora acentuada, ou alívio inicial da dor, em $94,9 \%$ dos casos, coincidindo com os achados da literatura ${ }^{31,85,111}$.

A rizotomia por radiofreqüência lesa seletivamente fibras $\mathrm{A} \delta$ e $\mathrm{C}$ regionais ${ }^{39}$, nessa seqüência, pois são mais sensíveis às lesões térmicas que promovem inativação de enzimas ou de sistemas enzimáticos específicos $^{51}$ que as fibras de grosso calibre ${ }^{5,19}$. Segundo Penman $^{68}$, foi Rathi, em 1913, quem, pela primeira vez, utilizou a eletrocoagulação dos ramos periféricos do nervo trigêmeo para tratar a dor facial. Kirschner ${ }^{42}$, em 1931, foi o primeiro a empregar a eletrocoagulação do gânglio de Gasser com a mesma finalidade e com o doente anestesiado sem controle intra-operatório de analgesia induzida, da qual resultaram numerosas complicações ${ }^{43}$. Thiry ${ }^{100}$, em 1962, empregou a eletrocoagulação controlada e obteve analgesia ou hipalgesia com preservação da sensibilidade táctil. White e Sweet ${ }^{113}$, em 1969, passaram a empregar geradores de radiofreqüência, eletródios providos de dispositivos que permitiram medir a temperatura e controlar a extensão da lesão, estimuladores elétricos para identificar o território do nervo trigêmeo e anestésicos de ação ultracurta para eliminar o desconforto do procedimento ${ }^{92,113}$. Petuisete e cols. ${ }^{69}$, em 1972, utilizaram eletródios bipolares, Lazorthes e cols. $^{48}$, em 1976, empregaram instrumental que permitia fixar e deslocar o eletródio micrometricamente, Tobler e cols. ${ }^{102}$, em 1983, descreveram eletródio com extremidade angulada com a finalidade de tornar o método mais seletivo, e Schürmann e cols., em 1972, empregaram a neuroleptoanalgesia para a eletrocoagulação parcial e seletiva do gânglio de Gasser, mantendo o doente desperto para possibilitar o controle intra-operatório da sensibilidade. Com as modificações propostas, a rizotomia percutânea, por radiofreqüência, tornou-se bastante divulgada nos centros neurocirúrgicos de destaque ${ }^{30,59,91,93,96}$. As dificuldades técnicas para punção ganglionar devidas à punção da artéria carótida ${ }^{94}$, a hematomas da face ${ }^{8} \mathrm{e}$ à presença da barra pterigo-alar ${ }^{61}$ ocorrem em menos $3,2 \%$ das vezes ${ }^{94}$ e não foram observadas quando da realização do presente trabalho.

Foi, propositadamente, induzida anestesia, pelo menos parcial, do território comprometido pela dor em $31,3 \%$ dos nossos casos, e analgesia em, pelo menos, uma divisão nos demais. $\mathrm{O}$ procedimento foi mais seletivo quando a lesão foi realizada com o doente desperto. Segundo Wepsic ${ }^{111}$, há necessidade da produção de intenso déficit sensitivo para que haja alívio da dor oncológica. A rizotomia por radiofreqüência, geralmente, proporciona analgesia ou hipalgesia e manutenção da sensibilidade táctil ${ }^{85}$ que podem ser parcialmente reversíveis ${ }^{4}$. Usualmente, há melhora do déficit sensitivo, semanas após o procedimento ${ }^{85,91}$. Raramente, o déficit acentua-se com o passar do tempo ${ }^{98}$. As complicações temporárias dos procedimentos ocorreram em $2,6 \%$ dos casos, cifras inferiores às relatadas em outros trabalhos. Ocorreram parestesias faciais discretas em dois $(5,1 \%)$ doentes. Alguns autores descreveram parestesias em $8 \%$ a $10,9 \%{ }^{30,59,98,101}$, disestesias dolorosas em $0,5 \%$ a $5 \%$ e anestesia dolorosa em menos de $5 \%{ }^{59,61,65,70,75,85,94,98,101,110}$ dos doentes com neuralgia do trigêmeo tratados com a técnica da rizotomia por radiofreqüência ${ }^{61,65,73,75,101,103}$. As anormalidades da sensibilidade causadas pela rizotomia são interpretadas como dormência nas primeiras semanas após a cirurgia $^{111}$. Com o passar do tempo, os doentes adaptamse a ela. A preservação do tato reduz o índice de anestesia dolorosa ou de disestesias. Entretanto, analgesia ou hipoestesia dolorosa ocorre em $0,1 \%$ a $1 \%$ dos doentes ${ }^{110,111}$. Ceratite ocorreu em um doente $(2,6 \%)$ e, úlcera de córnea, em outro $(2,6 \%)$ nesta casuística. A hipoestesia da córnea é observada em até $20 \%$ dos doentes ${ }^{61,65,86,101}$; muitas vezes, o reflexo corneopalpebral mantém-se presente, normal ou hipoativo ${ }^{30}$; quando hipoativo, torna-se normal com o passar do tempo. A ceratite ocorre em menos de 2,6\% dos $\operatorname{casos}^{30,59,85,87,98}$, a úlcera de córnea, em menos de $2 \%{ }^{30,33,59,86,87}$ e o comprometimento da visão ou cegueira, em menos em $4 \%{ }^{85,94}$. Com o uso de colírios, como realizado nos doentes da presente casuística, óculos especiais e tamponamento ocular é possível prevenir complicações sérias ${ }^{30,59}$. Ocorreu paresia temporária da motricidade ocular extrínseca em um $(2,6 \%)$ dos casos dessa casuística. Paralisia transitória da motricidade ocular extrínseca, especialmente do nervo troclear, é rara ${ }^{84,92,97,98,111}$. A lesão do nervo motor ocular comum e do nervo abducente ocorrem mais freqüentemente quando o eletródio penetra o seio cavernoso ${ }^{97}$. Paresia ou paralisia incapacitante do ramo motor do nervo trigêmeo, resultando em dificuldade para a mastigação e deglutição ${ }^{59,65,76,85,110,111}$ descritas freqüentemente por outros autores, foi observada em apenas 2,6\% doentes da presente casuística. Dor ou mal-estar no conduto auditivo, atribuídos à hipoestesia, sensação de barulho ou disacusia homolateral são atribuídos à disfunção do músculo tensor do tímpano ou à paresia do músculo tensor do véu palatino e à inadequada oclusão da trompa de Eustáquio ${ }^{94}$. Malestar nos olhos, caracterizado por lacrimejamento excessivo, ressecamento e prurido ocular ${ }^{94}$, lesão do nervo hipoglosso $0^{30,101}$, convulsão ${ }^{111}$, erupção pelo vírus do herpes simples ${ }^{91}$, hematoma intratemporal ${ }^{110}$, abscesso temporal ${ }^{11}$ e meningite asséptica ${ }^{91,111}$ relatados por outros autores não foram observados neste trabalho. Incapacidades devidas à hipoestesia lingual, 
dificuldade para adaptação à prótese dentária, hipersalivação, redução de produção de saliva, atrofia da mucosa nasal, aumento ou redução da secreção nasal não foram observados, talvez devido ao comprometimento funcional do nervo trigêmeo préoperatoriamente. A mortalidade é nula na maioria das casuísticas $^{30,70,85,94}$. Complicações clínicas intra-operatórias, como hipotensão arterial, arritmia cardíaca e precordialgias, ocorrem raramente ${ }^{106,110,111}$.

A taxa de recorrência observada de $7,7 \%$ é baixa ${ }^{31}$, porque, provavelmente, o período de acompanhamento dos nossos doentes foi curto. Houve recorrência da dor em 57\% dos casos acompanhados por Hitchcock e cols. ${ }^{31}$, em 1982, durante os primeiros 6 meses, sendo nova rizotomia necessária para aliviar a dor em $25 \%$ dos casos. Nos demais, a dor recorrente foi controlada com analgésicos. A reoperação foi indicada em $5,1 \%$ dos casos, devido à recorrência da dor. A recorrência da dor pode ser devida à progressão da doença ou à analgesia insatisfatória. A taxa de recorrência é maior quanto mais preservada estiver a sensibilidade da face ${ }^{94}$.

Houve necessidade da realização concomitante ou ulterior de rizotomia do nervo glossofaríngeo em 41,0\% dos doentes com dor no território de distribuição dessas estruturas nervosas. $\mathrm{O}$ tratamento neurocirúrgico da dor no território do nervo glossofaríngeo foi descrito, pela primeira vez, por Sicard e Rolineau ${ }^{83}$ que realizaram a secção das terminações periféricas do nervo glossofaríngeo em 1920. Adson ${ }^{1}$, em 1924, sugeriu a realização da rizotomia por via intracraniana. Essa técnica, desde então, é utilizada para o tratamento da dor oncológica orofaríngea ${ }^{71}$. Em 1948, Love realizou, pela primeira vez, a adição da secção intracraniana das fibras rostrais do nervo vago à do nervo glossofaríngeo por via intracraniana $a^{3,10,78}$. Entretanto, a freqüência de complicações e seqüelas, nos doentes operados pela rizotomia a céu aberto, é elevada e situada ao redor de $25 \%$. Por essa razão, Lazorthes e Verdie ${ }^{49}$, em 1979, descreveram a técnica de rizotomia percutânea por radiofreqüência do nervo glossofaríngeo. Esse procedimento revelou-se seguro e eficaz ${ }^{36,73}$, mesmo no tratamento da dor orofaríngea causada por neoplasias malignas $^{79}$.

A rizotomia espinal é, há muitos anos, empregada para o tratamento da dor nos doentes com câncer ${ }^{113}$. O procedimento percutâneo, desenvolvido por Uematsu e cols. ${ }^{105} \mathrm{em} \mathrm{1974}$, entretanto, é mais seguro e igualmente eficaz e foi aplicado em 16 casos dessa casuística.

Um doente apresentou, pós-operatoriamente, dor facial por desaferentação em conseqüência de neuropatia actínica e de neuropatia induzida pela rizotomia e dor no território do nervo glossofaríngeo e foi submetido, ulteriormente, à nucleotratotomia trigeminal estereotáxica. Serra e Negri ${ }^{82}$, em 1936, realizaram, pela primeira vez, a secção do trato espinal do nervo trigêmeo na região do ângulo pontocerebelar para o tratamento de dois doentes com dor causada por processos expansivos da base do crânio. Segundo Olivecrona $^{63}$, foi Sjöqvist, em 1937, quem realizou, pela primeira vez, a secção do trato descendente do nervo trigêmeo, na junção entre o terço médio e o terço inferior da oliva. Devido às complicações ${ }^{88}$, a técnica original foi aperfeiçoada ${ }^{16}$. Hosobuchi e Rutkin ${ }^{35}$, em 1971, recomendaram o emprego do potencial evocado intraoperatório para delimitar a área da lesão e utilizaram a radiofreqüência para produzir a lesão. Com a finalidade de promover analgesia nas porções centrais da face, $\mathrm{Kunc}^{46}$, em 1979, propôs a incisão vertical do trato e do núcleo do trato espinal do nervo trigêmeo. Grant e cols. ${ }^{22}$, em 1940, concluíram que a tratotomia a céu aberto era método excelente para o tratamento da dor oncológica. As taxas de morbidade e de mortalidade eram, entretanto, elevadas com tal procedimento. Ataxia, geralmente transitória ${ }^{16}$, nistagmo, disfagia, disartria ${ }^{74}$, disfonia, síndrome de Claude Bernard-Horner, hipoestesia contralateral, erupção pelo vírus do herpes simples ${ }^{16}$, abolição ou hipoatividade do reflexo corneopalpebral ${ }^{21}$ e soluços são comuns com o procedimento a céu aberto ${ }^{16}$. A ceratite ocorre excepcionalmente ${ }^{3,16,21,22,47,74,88}$. Disestesias, mas não anestesia dolorosa ${ }^{109}$, foram descritas em $6,4 \%$ a $100 \%$ dos casos $^{16,109}$. A mortalidade cirúrgica variou entre $0 \%$ e $16 \%{ }^{16,21,64,88}$. Com a finalidade de reduzir a freqüência de complicações próprias dos procedimentos a céu aberto e de destruir, com radiofreqüência, as unidades desaferentadas do núcleo do trato espinal do nervo trigêmeo, Crue e cols. ${ }^{12}$, em 1967, e Todd e cols. ${ }^{103}$, em 1969, descreveram a tratotomia trigeminal semi-esterotáxica, Hitchoock ${ }^{28}$, em 1970, a técnica estereotáxica e Fox ${ }^{18}$, em 1973, a técnica à mão livre. Este procedimento foi amplamente indicado para o tratamento da dor facial oncológica ${ }^{11,12,18,56,81}$ por apresentar a vantagem de aliviar a dor no território do nervo trigêmeo, intermediário e glossofaríngeo, freqüentemente presente em doentes com dor causada por processos neoplásicos orofaríngeos e cervicofaciais $^{11,29,81}$. Os resultados iniciais foram satisfatórios na maioria dos doentes ${ }^{11,29,80,81} \mathrm{e}$ a taxa de recorrência variou entre $15,2 \%$ e $25 \%{ }^{11,18,29,81}$. As complicações da nucleotratotomia espinal por radiofreqüência foram, geralmente, transitórias e consistiram em ataxia sensitiva ${ }^{18,80}$, soluços ${ }^{18} \mathrm{e}$ lesão do trato espinotalâmico ${ }^{18}$. $\mathrm{O}$ procedimento percutâneo tem a grande vantagem de poder ser realizado no doente consciente, possibilitando o controle do resultado do tratamento durante o ato operatório. Segundo Mazars e cols..$^{53}$, em 1976, a tratotomia pontina foi proposta por Dogliotti em 1938. Em 1987, Hitchcock e Teixeira ${ }^{32}$ descreveram a técnica da nucleotratotomia trigeminal pontina estereotáxica e recomendaram-na a doentes em que houve refratariedade da dor diante da nucleotratotomia espinal.

Outras técnicas foram descritas para o tratamento da dor facial e potencialmente são úteis para a dor gerada pelo câncer. Walker, em 1942, descreveu a técnica da mesencefalotomia a céu aberto. Spiegel e Wycis realizaram a primeira mesencefalotomia esterotáxica em $1947^{114}$. A mesencefalotomia e a talamotomia dos núcleos talâmicos inespecíficos passaram a ser bastante indicadas no tratamento da dor

Rizotomia percutânea para algias oncológicas —_ 199 
facial de natureza oncológica ${ }^{28,31,55,66,104,114}$. Entretanto, a recorrência do quadro álgico é comum ${ }^{66}$ e as complicações desses procedimentos freqüentes e, felizmente, transitórias ${ }^{31}$. A lobotomia e a leucotomia frontal ou a cingulotomia e outros procedimentos psicocirúrgicos são úteis para o tratamento da anestesia dolorosa quando há transtornos afetivos associados ${ }^{37,53,66}$. A ressecção cortical das áreas de projeções sensitivas da face fo, $22,77_{\text {não é mais empregada }}$ para o tratamento da dor. A estimulação elétrica do grupamento nuclear talâmico posterior foi pouco aplicada no tratamento da dor facial e os resultados proporcionados foram controversos ${ }^{54,90}$.

Conclui-se que a rizotomia percutânea do nervo trigêmeo do glossofaríngeo e das raízes cervicais é eficaz para o tratamento da dor orofaríngea decorrente de neoplasias malignas. A técnica é segura e de baixo custo operacional podendo ser indicada em doentes com afecções clínicas que contra-indiquem procedimentos de grande porte.

\section{Referências}

1. ADSON AW: The surgical treatment of glossopharyngeal neuralgia. Archs Neurol Psychiat 12:487-506, 1924.

2. ARNOULD G, LEPOIRE J, TRIDON P, LAXENAIRE M: Les neurinomes du trijumeau. Particularités cliniques et radiologiques. Rev Neurol (Paris) 104:302-8, 1961.

3. BOHM E, STRANG RR: Glossopharyngeal Neuralgia. Brain 85:371-88, 1962.

4. BRODKEY JS, MIYAZAKI Y, ERVIN FR: Reversible lesion with radiofrequency current. A method of stereotactic localization. J Neurosurg 21:49-53, 1964.

5. BROGGI G, SIEGFRIED J: The effect of graded thermocoagulation on trigeminal evoked potentials in the cat. Acta Neurochir (Wien) (suppl) 24:175-8, 1977.

6. BURCHIEL KJ, STEEGE TD, HOWE JF, LOESER JD: Comparison of percutaneous radiofrequency gangliolysis and microvascular decompression for the surgical management of tic doloureux. Neurosurgery 9:111-9, 1981

7. CANELAS HM, JULIÃO OT: Paralisias múltiplas de nervos encefálicos produzidas por neoplasias da base do crânio. Arq Neuropsiquiat (São Paulo) 19:185219, 1961

8. CHAKRAVORTY B: Visual disturbance following fifth nerve ganglion injection. Report of two cases. J Neurosurg 23:354-6, 1956.

9. CRAIG WM: Typical and atypical neuralgia of the face and neck. Proc Staff Meet Mayo Clin 11:677-81, 1936.

10. CHAWLA JC, FALCONER MA: Glossopharyngeal and vagal neuralgia. Br Med J 3:529-31, 1967.

11. CRUE BL, CARREGAL EJA, FELÖÖRY A: Percutaneous stereotact radiofrequency trigeminal tractotomy with neurophysiological recordings. Confin Neurol 34:389, 1972.

12. CRUE BL, TOOD EM, CARREGAL EJA, KILHAM O: Percutaneous trigeminal tractotomy. Case report utilizing stereotactic radiofrequency lesion. Bull Los Angeles Neurol Soc 32:86-92, 1967.

13. DANDY WE: The treatment of trigeminal neuralgia by the cerebellar route. Ann Surg 96:787-95, 1932.
14. DOTT NM: Facial pain. Proc Roy Soc Med 44:10347, 1951

15. ECKER A: Precise selective alcoholic Gasserian injection for tic doloureux. Recent advances in technic and results Acta Neurochir (Wien) 34:241-3, 1976.

16. FALCONER MA: Intramedullary trigeminal tractotomy and its place in the treatment of facial pain. J Neurol Neurosurg Psychiatry 12:297-311, 1949.

17. FOLEY KM: Pain syndrome in patients with cancer. In: Bonica JJ. Ventafrida V (ed): Advances in pain research and therapy. New York, Raven Press, 1979, vol 2, pp 59-75.

18. FOZ JL: Percutaneous trigeminal tractotomy for facial pain. Acta Neurochir (Wien) 26:83-8, 1973.

19. FRIGYESI TL, SIEGFRIED J, BROGGI G: The selective vulnerability of evoked potentials in the trigeminal sensory root to graded thermocoagulation. Exp Neurol 49:11-21, 1975.

20. GRANT FC: Results of the operative treatment of mayor trigeminal neuralgia. Ann Surg 107:14-9, 1938.

21. GRANT FC: Surgical methods for relief of pain. Bull NY Acad Med 19:373-85, 1943.

22. GRANT FC, GROFF RA, LEWY FH: Section of the descending spinal root of the fifth cranial nerve. Arch Neurol Psychiat (Chicago) 43:498-509, 1940.

23. HANKANSON S, LEKSELL L: Stereotatic gamma radiation in trigeminal neuralgia. In $6^{\text {th }}$ International Congress of Neurological Surgery. Amsterdam, Excerpta Medica, 1977, pp 57.

24. HALPERN LM: Psychotropics, ataractics and related drugs. In: Bonica JJ. Ventafrida (ed): Advances in pain research and therapy. New York, Raven Press, 1979, vol 2, pp 275-83.

25. HAMBY WB: Effectivenies of various operation for trigeminal neuralgia. J Neurosurg 17:1039-44, 1960.

26. HANRAETS PR: Surgical treatment of trigeminal neuralgia following the method of Dandy. Some historical remarks and technical suggestions. Psychiatr Neurol Neurochir 73:441-6, 1970.

27. HÄRTEL F: Röentgenologische Darstellung das Foramen Ovale des Schädels und ihre Bedertung für die Behandlunmg der Trigeminus neuralgie. Dtsch Med Wschr 61:1069-72, 1935.

28. HITCHCOCK E: Stereotactic trigeminal tractotomy. Ann Clin Res 2:131-5, 1970.

29. HITCHCOCK E: Stereotactic spinal surgery. In: Carrea R. Levy D (ed): Neurological Surgery International Congress Series $n^{\circ} 433$. Amsterdam, Excepta Medica, 1977, pp 271-80.

30. HITCHOCOCK E, TEIXEIRA MJ: A comparison of results from center-median and basal thalamotomies for pain. Surg Neurol 15:341-51, 1981.

31. HITCHOCOCK H, TEIXEIRA M, PINTO J: Percutaneous trigeminal radiofrequency rhyzotomy. J Royal Coll Surg Edinburgh 28:747-9, 1983.

32. HITCHCOK ER, TEIXEIRA MJ: Pontine stereotactic surgery and facial nociception. Neurobiol Res 9:1137, 1987.

33. HORRAX G: The role of minor procedures in the treatment of trigeminal neuralgia. Surg Clin North Am 26:774-9, 1976

34. HORTON J, MEANS ED, UNNINGHAM RJ, OLSON KB: The numb chin in breast cancer. J Neurol Neurosurg Psychiatry 36:211-6, 1973.

35. HOSOBUCHI Y, RUTKIN B: Descending trigeminal tractotomy. Neurophysiological approach. Arch Neurol 25:115-25, 1971

36. ISAMAT F, FERRAN E, ACEBES JJ: Seletive percutaneous thermocoagulation rhizotomy in 
essential glossopharyngeal neuralgia. J Neurosurg 55: 575-80, 1981.

37. JAEGER R: The resuslts of injecting hot water into the Gasserian ganglion for the relief of tic doloureux. J Neurosurg 16:656-63, 1959.

38. JANJIGIAN ER: Trigeminal neuralgia apparently cured by electro shok therapy; 3 cases. Am J Psychiatry 106:143-6, 1948.

39. JANNETTA PJ, RAND RW: Transtentorial retrogasserian rhizotomy in trigeminal neuralgia. In: Rand RW (ed): Microsurgery. St Louis, CV Mosby, 1969, pp 156-169.

40. JEFFERSON A: Trigeminal root and ganglion injections using phenol in glycerine for the relief of trigeminal neuralgia. J Neurol Neurosurg Psychiatry 26:345-52, 1963.

41. KING RB, MEAGHER JM: Studies of trigeminal nerve potencials. J Neurosurg 12:393-402, 1955

42. KIRSCHNER M: Zur Elektrochirurgie. Arch Klin Chir 167:761-8, 1931

43. KIRSCHNER M: Zur Elektrokoagulation das Ganglion Gasseri. Zbl Chir 59:2841-2, 1932.

44. KIRSCHNER M: Zur Behandlung der Trigeminusneralgie. Med Wochenschr (Munich) 89:235-9, 1974.

45. KUBANYI A: Sur le traitement de la névralgie du nerve trijumeau para l'électrocoagulation intracranianne du ganglion de Gasser (301 cas). Lyon Chir 41:681-9, 1946.

46. KUNK Z: Vertical trigeminal partial nucleotomy. In: Bonica JJ. Liebeskind JC. Albe-Fessard DG (ed): Advances in pain research and therapy. New York, Raven Press, 1979, vol 3, pp 325-330.

47. LAZORTHES G, ESPAGNO J, LACOMBE Y: Competion nouvelle de la névralgie trigeminale esséntielle. Mem Acad Chir (Paris) 94:286-290, 1968.

48. LAZORTHES G, VERDIE JC, BOUYSSOUR M: Intérét de l'utilisation d'um cadrè "stéreotaxique" dans la thermocoagulation sélective du ganglion de Gasser. Neurochirurgie 22:77-83, 1976.

49. LAZORTHES Y, VERDIE JC: Radiofrequency coagulation of the petrous ganglion in glossopharyngeal neuralgia. Neurosurgery 4:512-6, 1979.

50. LE BEAU J, DAUM S, FORJAZ S: Trigeminal tractotomy in the treatment of facial neuralgias. Year Book of Neurol Psychiat \& Neurosurg. The Year Book Publishers Inc, Chicago, 1949, pp 620.

51. LETCHER FS, GOLDRING S: The effect of radiofrequency current and heat on peripheral nerve potential in the cat. J Neurosurg 29:42-7, 1968.

52. MARKHAN JW: Sudden loss of vision following alcohol block of the infraorbital nerve: case report. J Neurosurg 38:655-7, 1973.

53. MAZARS G, MERIENNE L, CIOLOCA C: État actual de la chirurgie de la douleur. Neurochirurgie 22 (Suppl 1): 5-164, 1976.

54. MAZARS G, MERIENNE L, CIOLOCA C: Comparative study of electrical stimulation of posterior thalamic nuclei, periaqueductal grey and other medline mesencephalic structures in man. In: Bonica JJ. Liebeskind JC. AlbeFessard DG (ed): Advances in pain research and therapy. New York, Raven Press, 1979, vol 3, pp 541-6.

55. MAZARS G, ROGE R, PANSINI A: Stereotactic coagulation of the spinothalamic tract for intractable trigeminal pain. J Neurol Neurosurg Psychiatry 23:352-60, 1960.

56. MEHTA DS, MALIK GB, PATH MC, DAR J: Trigeminal neuralgia due to cholestectomia of Meckel's cave report. J Neurosurg 34:572-4, 1971.

57. MORELLO G, BIANCHI M: Considerazioni su 449 casi di nevralgia trigeminale curati chirurgicamente. Minerva Neurochir 39:103-4, 1966.
58. MORLEY TP: Summary of panel on controversies in the management of trigeminal neuralgia. Clin Neurosurg 24:584-9, 1976.

59. MOTTA P, DE SOUZA MTK, SENGUPTA RP: Radiofreqüência - termocoagulação no tratamento da neuralgia do trigêmeo. Análise de 100 casos. Arq neuropsiquiatr (São Paulo) 38:33-44, 1980.

60. MURPHY JB: Osmic acid injections for relief of trifacial neuralgia. JAMA 43:947-55, 1904.

61. O'CONNEL JEA: Trigeminal false localizing signs and their causation. Brain 101:119-42, 1978.

62. ODOM GL, LYMAN PS: Speach disorder following excision of post-central gyrus. Trans Am Neurol Ass 71:67-70, 1946

63. OLIVECRONA H: The syndrome of painful anesthesia following section of the sensory root of the fifth nerve in tic doloureux. Acta Chir Scand 82:99-106, 1939.

64. OLIVECRONA H: La cirurgia del dolor. Arch Neurocirurg 4:1-10, 1947.

65. ONOFRIO BM: Radiofrequency percutaneous Gasserian ganglion lesions. Results in 140 patients with trieminal pain. J Neurosurg 42:132-9, 1975.

66. ORTHNER KH, ROEDER F: Further clinical and anatomical experience with stereotactic operations for relief of pain. Confin Neurol 27:418-30, 1966.

67. PENMAN J: Some development in the technique of trigeminal injection. Lancet 1: 760-4, 1953.

68. PENMAN J: Trigeminal neuralgia. In: Vinken PJ. Bruyn GW (ed): Handbook of Clinical Neurology. Amsterdam, North-olland Publishing Company, 1968, vol 5, pp 296-332.

69. PERTUISET B, PHILIPPON J, FOHANNO D, KHALIL $M$ : Traitment microchirurgical de la névralgie faciale essentialle par neurotomie rétrogassérienne sélective transtentorielle. Rev Neurol (Paris) 126:97106, 1972.

70. PERTUISET B, PHILIPPON J, MACHANAKIAN A: Électrothermie rétro-gasseriene dans la névralgie faciale. Ann Med Int (Paris) 126:657-60, 1975.

71. PEET MM: Glossopharyngeal neuralgia. Ann Surg 101:256-68, 1935.

72. PUTNANN TJ, HAMPTON AO: A technic of injection into the Gasserian ganglion under roentgenographic control. Arch Neurol Psychiat (Chicago) 35:92-8, 1936.

73. RAND RW: Gardner neurovascular decompression of the trigeminal and facial nerves for tic doloureux and hemifacial spasm. Surg Neurol 16:329-32, 1981.

74. RANEY R, RANEY AA, HUNTER CR: Treatment of major trigeminal neuralgia through section of the trigeminospinal tract in the medulla. Am J Surg 80:117, 1950.

75. RISH BL: Cerebrovascular acident after percutaneous of thermocoagulation of the trigeminal ganglion. Case report. J Neurosurg 44:376-7, 1976.

76. ROTHMAN KJ, WEPSIC JC: Side of facial pain in trigeminal neuralgia. J Neurosurg 40:514-6, 1974.

77. RUGE D, BROCHNER R, DAVIS L: A study of the treatment of 637 patients with trigeminal neuralgia. J Neurosurg 15:528-36, 1958.

78. RUSHTON JG, STEVENS JC, MILLER RH: Glossopharyngeal (vagoglopharungeal) Neuralgia. Arch Neurol 38:201-5, 1981.

79. SCHIBUOLLA CT: Resultados imediatos do tratamento cirúrgico da nevralgia do glossofaríngeo. Dissertação de mestrado. Faculdade de Medicina da Universidade de São Paulo, 1982.

80. SCHVARCZ JR: Stereotactic trigeminal tractotomy. Confin Neurol 37:73-7, 1975. 
81. SCHVARCZ JR: Spinal cord techniques: trigeminal nucleotomy and extralemniscal myelotomy. Appl Neurophysiol 41:99-112, 1978.

82. SERRA A, NERI V: Die elektro-chirurgische Unterbreching der Zentralbaohner des V. paares an lateralen ventralen Rand des Pons Varoli als erster Behendlungsversuch von hartnackingen Neuralgien des Trigeminus durch Yomren der Schödelbasis. Zbl chir 63:224-8, 1936.

83. SICARD R, ROBINEAU V: Algie velo-pharyngée essentielle. Traitment chirurgical. Rev Neurol 36:2567, 1920.

84. SIEGFRIED J: Un nouveau traitiment neurochirurgical de la névralgie du trijumeau: analgésie sans anésthésie. Neurochirurgie 19:585-7, 1973.

85. SIEGFRIED J: 500 percutaneous thermocoagulation of the Gasserian ganglion for trigeminal pain. Surg Neurol 8:126-31, 1977.

86. SINDOU M: Thermocoagulation percutanée du trijumeau dans le traitment de la névralgie faciale essentialle. Résultats en function du siège de la thermolésion. Neurochirurgie 25:166-72, 1979.

87. SINDOU M, KERAVEL Y: La thermocoagulation percutanée du trijumeau. Noveau traitment neurochirurgical de la névralgie faciale essentialle. Nouv Press Med 5:1583-4, 1976.

88. SJÖQVIST D: Trigeminal neuralgia. Review of its surgical treatment and some aspects of its etiology. Acta Chir Scand 82:201-17, 1939.

89. STAMMERS FAR: A study of tumours and inflammations of the Gasserian ganglion. Br J Surg 18:125-153, 1930.

90. STRASBURG HM, THODEN $V$, MUNDINGER F: Mesencephalic chronic electrodes in pain patients. An electrophysiological study. Appl Neurophisiol 42:248-93, 1979.

91. SWEET WH: Treatment of facial pain by percutaneous differential thermal trigeminal rhizotomy. Prog Neurol Surg 7:153-79, 1976.

92. SWEET WH, MARK VH, HAMLIN H: Radiofrequency lesions in the central nervous system of man and cat, including case reports of eight bulbar pain tract interruptions. J Neurosurg 17:213-25, 1960.

93. SWEET WH, WEPSIC JG: Relation of fiber size in trigeminal posterior root to conduction of impulses for pain and touch. Production of analgesia without anesthesia in the effective treatment of trigeminal neuralgia. Trans Am Neurol Assoc 95:134-9, 1970.

94. SWEET WH, WEPSIC JG: Controled thermocoagulation of trigeminal ganglion and rootlets for differential destruction of pain fibers. 1. Trigeminal neuralgia. $J$ Neurosurg 40:143-56, 1974.

95. TEIXEIRA MJ: A rizotomia por radiofreqüência e a descompressão neurovascular do nervo trigêmeo no tratamento das algias da face. Tese (Mestrado). Universidade de São Paulo, 1985, pp 256.

96. TEW JM Jr, KELLER JT: The treatment of trigeminal neuralgia by percutaneous radiofrequency technique. Clin Neurosurg 12:557-578, 1976.

97. TEW JM Jr, KELLER JT, WILLIAMS DS: Application of stereotactic principles to the treatment of trigeminal neuralgia. Appl Neurophysiol 41:146-56, 1978.

98. TEW JM Jr, MAYFIELD FH: Trigeminal neuralgia. A new surgical approach (percutaneous electrocoagulation of the trigeminal merve). Laryngoscope 83:1096-101, 1973.
99. TEW JM: Treatment of pain of glossopharingeal and vagus nerves by percutaneous rhizotomy. In: Youmans JR (ed): Neurological Surgery. New York, Saunders, 1982, pp 3609-3612.

100. THIRY S: Expérience personelle basée sur 225 cas de névralgie essentielle du trijumeau traités par électrocoagulation stéreotaxique du ganglion de Gasser entre 1950 et 1960. Neurochirurgie 8:86-92, 1962.

101. THIRY S, ROTHERMANS JM: Traitment de la névralgie essentialle du trijumeau par stéreotaxie et electrocoagulation partielle sélective du ganglion de Gasser. Expérience portant sur 365 cas traités entre 1950 et 1970. Neurochirurgie 20:55-60, 1974.

102. TOBLER WD, TEW JM, COSMAN E, KELLER JT, QUALLEN B: Improved outcome in the treatment of trigeminal neuralgia by percutaneous stereotactic rhizotomy with a new curved tip electrode. Neurosurgery 12:313-7, 1983.

103. TOOD EM, CRUE BL, CARREGAL EJA: Posterior percutaneous tractotomy and cordotomy. Confin Neurol 31:106-15, 1969.

104. TSUBOKAWA T, MORIAYSU M: Follow-up results of centre median thalamotomy for relief of intratable pain. A method of evaluation the effectiveness during operation. Confin Neurol 37:280-4, 1965.

105. UEMATSU S, UDVARHELY JB, BERSON DW, SIEBENS AA: Percutaneous radiofrequency rhizotomy. Surg Neurol 2:319-24, 1974.

106. VER BRUGGHEN A: Paragasserian tumors. J Neurosurg 9:451-60, 1952.

107. VISCA A, BERNASCONI A: A radiographic technique for trigeminal neurolythic injections. Acta Neurochir (Wien) 2:55-60, 1972

108. WALKER E, MILES FC, SIMPSON RJ: Partial trigeminal rhizotomy using suboccipital approach. Experience in the treatment of trigeminal neuralgia. Arch Neurol Psychiatry 75:514-21, 1956.

109. WEINBERGER LM, GRANT FC: Experiences with intramedullary tratatomy. III. Studies in sensation. Arch Neurol Psychiat (Chicago) 48:355-81, 1942.

110. WEPSIC JG: Complications of percutaneous surgery for pain. Clin Neurosurg 83:454-64, 1975.

111. WEPSIC JG: Technique for radiofrequency gasserian ganglionectomy. Appl Neurophysiol 39:122-32, 1976.

112. WHISLER WW, VORIS HC: Mesencephalotomy for intractable pain due to malignant disease. Appl Neurophysiol 41:52-6, 1978.

113. WHITE JC, SWEET WH: Pain and the neurosurgeon. Springfield, Illinois, Charles C. Thomas Publisher, 1969.

114. WYCIS HT, SPIEGEL EA: Long range results in the treatment of intractable pain by stereotactic midbrain surgery. J Neurosurg 19:101-7, 1962.

Original recebido em abril de 1999

Aceito para publicação em julho de 1999

\section{Endereço para correspondência:}

Manoel Jacobsen Teixeira

Rua General Mena Barreto, 756

CEP 01433-010 - São Paulo, SP 